TIMP-1 is significantly associated with objective response and survival in metastatic colorectal cancer patients receiving combination of irinotecan, 5-fluorouracil, and folinic acid

Sørensen, Nanna Møller; Byström, Per; Christensen, lb J.; Berglund, Åke; Nielsen, Hans Jørgen; Brünner, Nils; Glimelius, Bengt

Published in:

Clinical Cancer Research

DOI:

10.1158/1078-0432.CCR-07-0186

Publication date:

2007

Document version

Publisher's PDF, also known as Version of record

Citation for published version (APA):

Sørensen, N. M., Byström, P., Christensen, I. J., Berglund, Å., Nielsen, H. J., Brünner, N., \& Glimelius, B. (2007). TIMP-1 is significantly associated with objective response and survival in metastatic colorectal cancer patients receiving combination of irinotecan, 5-fluorouracil, and folinic acid. Clinical Cancer Research, 13(14), 4117-4122. https://doi.org/10.1158/1078-0432.CCR-07-0186 


\title{
TIMP-1 Is Significantly Associated with Objective Response and Survival in Metastatic Colorectal Cancer Patients Receiving Combination of Irinotecan, 5-Fluorouracil, and Folinic Acid
}

\author{
Nanna M. Sørensen, ${ }^{1}$ Per Byström, ${ }^{2}$ lb J. Christensen, ${ }^{3} \AA^{\circ k e}$ Berglund, ${ }^{4}$ Hans Jørgen Nielsen, ${ }^{3}$ \\ Nils Brünner, ${ }^{1}$ and Bengt Glimelius ${ }^{2,4}$
}

\begin{abstract}
Purpose: Tissue inhibitor of metalloproteinase-1 (TIMP-1) is known to protect cells against apoptosis. We raised the hypothesis that elevated tumor tissue levels and thereby plasma levels of TIMP-1 would predict resistance to apoptosis-inducing chemotherapy.

Experimental Design: Ninety patients with metastatic colorectal cancer were included in the study. Plasma TIMP-1 and serum carcinoembryonic antigen (CEA) were measured in samples obtained before the first cycle of chemotherapy.

Results: Analysis of best objective response (complete or partial response versus stable or progressive disease) showed that patients with low plasma TIMP-1 had higher probability of obtaining an objective response [odds ratio (OR), 3.5; 95\% confidence interval $(95 \% \mathrm{Cl})$, 1.4-8.5, $P=0.007]$. CEA treated as a continuous variable was also a statistically significant predictor of no response $(\mathrm{OR}, 1.3 ; 95 \% \mathrm{Cl}, 1.0-1.7, P=0.02$, area under the curve 0.66$)$ but much less so. Plasma TIMP-1 was the only significant covariate in a multivariable analysis of best objective response (OR, 3.6; $95 \% \mathrm{Cl}, 1.4-9.5 ; P=0.001)$. PlasmaTIMP-1 scored as a continuous variable on the $\log$ scale $\left(\log _{\mathrm{e}}\right)$ was significantly associated with overall survival [OS; hazard ratio (HR), 3.8; 95\% Cl, 2.4-5.9; $P<0.0001]$ and with time to progression (TTP; HR, 1.5; 95\% Cl, 1.0-2.3; $P=$ 0.048). Multivariable analysis showed that plasmaTIMP-1 was significant for OS when including routine clinical baseline covariates ( $\mathrm{HR}, 3.5 ; 95 \% \mathrm{Cl}, 2.1-5.8 ; P<0.0001)$. A multivariable analysis including TTP instead of OS showed that only plasma TIMP-1 was retained in the model (HR, 1.5). CEA was not significantly associated with TTP or OS when TIMP-1 was included in the model. Conclusion: This study shows that plasma TIMP-1 levels are significantly and independently associated with objective response, TTP, and OS in patients with metastatic colorectal cancer receiving combination chemotherapy.
\end{abstract}

Authors' Affiliations: 'Section of Biomedicine, Department of Veterinary Pathobiology, Faculty of Life Sciences, University of Copenhagen, Copenhagen, Denmark; ${ }^{2}$ Department of Oncology and Pathology, Karolinska Institutet, Stockholm, Sweden; ${ }^{3}$ Department of Surgical Gastroenterology, Hvidovre University Hospital, Hvidovre, Denmark; and ${ }^{4}$ Department of Oncology, Radiology and Clinical Immunology, Akademiska sjukhuset, Uppsala University, Uppsala, Sweden Received 1/23/07; revised 4/18/07; accepted 5/10/07.

Grant support: The Danish and Swedish Cancer Societies, The IMK Foundation, The Danish Medical Research Counsel, Familien Hede Nielsens Fund, Beckett Foundation, The Danish Cancer Research Foundation, The Stockholm Cancer Society, Eva og Henry Frnkels Foundation, Grosserer Valdemar Foersom og Hustru Thyra Foersoms Foundations, Ib Henriksens Foundation, Kathrine og Vigo Skovgaard Foundation, Knud og Dagny Gad Andresens Foundation, Redaktør Kaaresens Foundation, The Obel Family Foundation, The Kornerup Fund, The Aage and Johanne Louis-Hansen Fund, The Aase and Einar Danielsens Fund, and P.A. Messerschmidt og Hustrus Foundation.

The costs of publication of this article were defrayed in part by the payment of page charges. This article must therefore be hereby marked advertisement in accordance with 18 U.S.C. Section 1734 solely to indicate this fact.

Note:The results of this study was presented at the 2006 AACR Meeting as a poster N.M. Sørensen and P. Byström contributed equally as first authors and B. Glimelius and N. Brünner contributed equally as senior authors to the scientific work.

Requests for reprints: Nils Brünner, Section of Biomedicine, Department of Veterinary Pathobiology, Faculty of Life Sciences, University of Copenhagen, Ridebanevej 3, 1870 Frederiksberg, Denmark. Phone: 45-26144708; Fax: 4535353514; E-mail: nbr@life.ku.dk.

(C) 2007 American Association for Cancer Research

doi:10.1158/1078-0432.CCR-07-0186
In Europe and the United States, the number of new colorectal cancer cases approximates 500,000 on a yearly basis. ${ }^{5}$ Approximately half of the patients will develop metastatic colorectal cancer disease, and these patients are candidates for systemic chemotherapy. However, the objective response rate [complete response (CR) and partial response (PR)] is only $\sim 50 \%$, which means that half of the patients will receive treatment with no substantial clinical benefit.

Today, there is no single biomarker that can be used to predict response/resistance to chemotherapy in metastatic colorectal cancer patients (1). As most types of conventional chemotherapy kill cancer cells by inducing apoptosis, it could be hypothesized that markers of apoptosis protection would be useful as markers of chemotherapy resistance.

The proteinases inhibitor, tissue inhibitor of metalloproteinase-1 (TIMP-1), inhibits, in addition to its matrix metalloproteinase inhibitory function, apoptosis $(2-5)$.

We and others have shown that plasma levels of TIMP-1 in patients with primary colorectal cancer are associated with patient outcome; that is, high plasma TIMP-1 predicts shorter patient survival $(6-12)$. One explanation to this association is

\footnotetext{
${ }^{5}$ www-dep.icrf/.fr
} 
that TIMP-1 protects cancer cells against the apoptotic stimuli that constitutively affect the cells. In support of an antiapoptotic function of TIMP-1, we have recently shown that in a cohort of 173 patients with metastatic breast cancer, high tumor tissue levels of TIMP-1 were highly significantly associated with chemotherapy resistance (13). For example, in patients with high tumor tissue TIMP-1 levels, the objective response (CR and $\mathrm{PR}$ ) to chemotherapy was $0 \%$. In accordance with these findings, Lipton et al. (14) showed that high plasma TIMP-1 was significantly associated with resistance to the antiestrogen tamoxifen in patients with metastatic breast cancer.

Based on the current knowledge of TIMP-1 as a protector of cancer cell apoptosis, we raised the hypothesis that levels of plasma TIMP-1 in patients with metastatic colorectal cancer would be predictive of response to chemotherapy. Indeed, the present study, including a homogeneous group of previously untreated patients treated with combination chemotherapy within a prospective trial, supports our hypothesis because a highly significant correlation was found between these two variables.

\section{Patients and Methods}

Patients and treatment. The study included 90 patients diagnosed with metastatic colorectal cancer. The study was done on a subpopulation of a larger study, NORDIC VI, a randomized phase III Nordic multicenter trial with 567 included patients comparing irinotecan in combination with either the Nordic bolus 5-fluorouracil (5-FU) and folinic acid schedule or the bolus/infused de Gramont schedule, in patients with metastatic colorectal cancer. All patients in two of the centers, Karolinska University Hospital (Stockholm, Sweden) and Akademiska Hospital (Uppsala, Sweden), were consecutively asked if they accepted the additional blood samples required in the study. In these two centers, 122 patients were randomized to the main study and 90 accepted the extra investigations on the blood samples. Of these, 87 were evaluable for objective response to chemotherapy.

Of the 90 patients, 77 had liver metastases, 23 lung metastases, 9 lymph node metastases, and 11 patients had multiple metastases.

Eligibility criteria included histologically proven adenocarcinoma of colon or rectum with metastatic disease, age between 18 and 75 years, no prior chemotherapy or only adjuvant chemotherapy if terminated $>6$ months before randomization was accepted, performance status (WHO) of 0 to 2, and at least one measurable metastatic lesion according to the Response Evaluation Criteria in Solid Tumors (15). Written informed consent was obtained for all patients. Exclusion criteria included central nervous system metastases, current infection, diabetes, unresolved bowel obstruction, uncontrolled inflammatory bowel disease, current history of chronic diarrhea, or any other serious medical condition.

According to the study protocol, patients were randomized to receive either of the following treatments:

Arm A. Irinotecan: $180 \mathrm{mg} / \mathrm{m}^{2}$ as a 30 to $90 \mathrm{~min}$ i.v. infusion on day 1 every 2 weeks immediately followed by 5-FU: $500 \mathrm{mg} / \mathrm{m}^{2}$ i.v. bolus on days 1 and 2 every 2 weeks, followed 30 to 40 min later by folinic acid: $60 \mathrm{mg} / \mathrm{m}^{2}$ i.v. bolus on days 1 and 2 every 2 weeks.

Arm B. Irinotecan: $180 \mathrm{mg} / \mathrm{m}^{2}$ as a 30 to $90 \mathrm{~min}$. i.v. infusion day 1 every 2 weeks, followed by folinic acid: $200 \mathrm{mg} / \mathrm{m}^{2}$ as a $2 \mathrm{~h}$ i.v. infusion, days 1 and 2 every 2 weeks, followed by 5 -FU: $400 \mathrm{mg} / \mathrm{m}^{2}$ i.v. bolus followed by $600 \mathrm{mg} / \mathrm{m}^{2}$ 5-FU as a $22 \mathrm{~h}$ i.v. infusion days 1 and 2 every 2 weeks.

Response evaluation. The primary efficacy variable was TTP defined as the time from randomization to the first documented progression or death due to progression. The secondary efficacy variable was overall survival (OS) calculated from the date of randomization until death. Responses were evaluated after every fourth cycle or every 2nd month if treatment was terminated. Treatment continued until progression or severe toxicity was noted. Overall response rate was defined as CR and $\mathrm{PR}$ as it is defined according to the Response Evaluation Criteria in Solid Tumors (15).

TIMP-1 analyses. EDTA plasma samples were drawn before start of chemotherapy. The samples were stored at $-80^{\circ} \mathrm{C}$ until analyzed. In addition, in 75 of the patients, plasma samples were collected at 2, 4, and 6 weeks of treatment (samples were drawn before the initiation of each cycle). As previously described, plasma levels of TIMP-1 were determined by use of an established, validated TIMP-1 ELISA (6). In brief, for measurements of TIMP-1 protein levels, microtiter plates were coated with a sheep polyclonal antibody and detection of bound TIMP1 (free and complex-bound forms) was done by use of a monoclonal antibody (MAC 15) and a secondary alkaline phosphatase-coupled antibody (DAKO). Readings of color development were taken every $10 \mathrm{~min}$ for $1 \mathrm{~h}$, and calculations of concentrations were based on included recombinant TIMP-1 protein standards.

Carcinoembryonic antigen analyses. Serum samples were drawn before start of chemotherapy and after 2, 4, and 8 to 10 weeks of treatment. The level of carcinoembryonic antigen (CEA) was measured using the immunometric assay from DiaSorin.

Statistics. Descriptive statistics are presented by the median and range. Tests for location were done using the Wilcoxon rank sum test or the Kruskal-Wallis test and the Spearman rank correlation was used as a measure of association. Covariates are either dichotomized by their median or entered by the actual value on the log scale (natural). Survival probabilities for time to progression (TTP) and OS are estimated by the Kaplan-Meier method and tests for differences between strata are done using the log-rank statistics. Analyses of TTP and OS for continuous covariates as well as multivariate analyses are done using the Cox proportional hazards model. The estimation of the probability of response is done using logistic regression analysis and results are presented using the odds ratio (OR) and the receiver operating characteristic curve, including the area under the curve. $P$ values $<5 \%$ are considered statistically significant. All calculations are done using SAS (version 9.1, SAS Institute).

\section{Results}

Levels of plasma TIMP-1 and association with baseline variables. All plasma samples contained measurable levels of TIMP-1. The median concentration was $121.9 \mathrm{ng} / \mathrm{mL}$ (range, 20.2-1,001.4 ng/mL) of plasma. The 95 percentile was 169.2 $\mathrm{ng} / \mathrm{mL}$ of TIMP-1. Associations between plasma TIMP-1 and baseline variables are given in Table 1. Plasma TIMP-1 values were significantly associated with performance status $(P=0.003)$. In addition, TIMP-1 marginally associated with adjuvant treatment $(P=0.049)$; high TIMP-1 levels were found in those patients who had not received adjuvant treatment. No significant associations were seen between plasma TIMP-1 and age $(r=0.16, P=0.12)$, gender $(P=0.36)$, location of the cancer (colon or rectum; $P=0.69$ ), or allocated treatment $(P=0.92)$.

Levels of plasma TIMP-1 and serum CEA and association with best objective response. Eighty-seven patients were evaluable for response. Analysis of best objective response was scored as CR or PR versus stable disease (SD) or progressive disease (PD). Four patients obtained CR, $36 \mathrm{PR}, 38 \mathrm{SD}$, and $9 \mathrm{PD}$. Of the 38 SD patients, eight had duration of $\mathrm{SD}<4$ months. When TIMP-1 was treated as a continuous variable, the univariable analyses showed that patients with high plasma TIMP-1 had higher probability of no response [SD or PD; OR, 3.5; $95 \%$ 
confidence interval, $(95 \% \mathrm{CI}), 1.4-8.5 ; P=0.007]$. The area under the curve was 0.67 . Using the median TIMP-1 levels to dichotomize the patients showed that among the 44 patients with low TIMP-1, 26 (59\%; 95\% CI, 44-74\%) obtained CR or $\mathrm{PR}$, whereas among the 43 patients with high plasma TIMP-1, only 14 (33\%; 95\% CI, 19-47\%) obtained an objective response. Stratifying patients with SD by duration of response showed that among the eight patients with $\mathrm{SD}<4$ months, seven $(88 \%)$ had plasma TIMP-1 levels above the median.

Plasma TIMP-1 scored as a continuous covariate on the log scale entered into a multivariate logistic regression analysis showed that only TIMP-1 was a significant predictor of no response (SD and PD; OR, 3.6; 95\% CI, 1.4-9.5; $P=0.001$; Table 2). The OR for a patient with TIMP-1 level equal to the 3rd quartile of TIMP-1 levels compared with one with TIMP-1 equal to the 1st quartile was 2.4 (95\% CI, 1.2-4.6).

Serum CEA levels were available for 67 of the 87 patients. The median CEA level was 56 units/mL, ranging from 1 to 4,073 . The rank correlation between CEA and TIMP-1 was 0.31 $(P=0.01)$. This subset of patients is similar to the entire data set with respect to the distribution of the clinical covariates, age, and TIMP-1 levels. CEA treated as a continuous variable was a significant predictor of no response (OR, 1.3; 95\% CI, 1.0-1.7; $P=0.02$; area under the curve 0.66). Combining CEA with TIMP-1 did not result in a significantly improved fit; the $P$ value to include CEA in a model with TIMP-1 was 0.09 .

Levels of plasma TIMP-1 and serum CEA and association with TTP and OS. Ninety patients were evaluable for TTP and OS. There was a significant trend between TTP [hazard ratio (HR), 1.5; 95\% CI, 1.0-2.3, $P=0.048$ ] and plasma TIMP-1 scored as a continuous variable on the log scale $\left(\log _{e}\right)$. Kaplan-Meier estimates of TTP are shown in Fig. 1 with TIMP-1 dichotomized by the median. The figure illustrates that patients with plasma TIMP-1 levels below the median have a significantly longer TTP (HR, 1.9; 95\% CI, 1.2-3.0; $P=0.01$ ) than patients with plasma

Table 1. Associations between plasma TIMP-1 and baseline covariates

\begin{tabular}{|c|c|c|}
\hline Covariate & $N=90$ & $\begin{array}{l}\text { Association to } \\
\text { TIMP-1 P }\end{array}$ \\
\hline \multicolumn{3}{|l|}{ Randomized to } \\
\hline FLIRI & 44 & 0.92 \\
\hline FOLFIRI & 46 & \\
\hline \multicolumn{3}{|l|}{ Gender } \\
\hline Male & 60 & 0.36 \\
\hline Female & 30 & \\
\hline \multicolumn{3}{|l|}{ Localization } \\
\hline Colon & 59 & 0.69 \\
\hline Rectum & 31 & \\
\hline \multicolumn{3}{|l|}{ Performance status WHO } \\
\hline 0 & 69 & 0.003 \\
\hline 1 & 17 & \\
\hline 2 & 4 & \\
\hline \multicolumn{3}{|l|}{ Adjuvant therapy } \\
\hline No & 79 & 0.049 \\
\hline Yes & 11 & \\
\hline Age median ( $\min , \max ; y)$ & $60(36-75)$ & $0.16 *$ \\
\hline
\end{tabular}

Abbreviations: FLRI, Nordic bolus 5-FU and folinic acid schedule; FOLFIRI, bolus/infused de Gramont schedule.

*Spearman rank correlation $(P=0.12)$.
Table 2. Multivariate logistic regression analysis of no response (SD and PD) to chemotherapy

\begin{tabular}{|c|c|c|}
\hline Covariate & $\boldsymbol{P}$ & OR (95\% CI) \\
\hline \multicolumn{3}{|c|}{ Randomized to } \\
\hline FLIRI & 0.54 & $1.3(0.5-3.5)$ \\
\hline FOLFIRI & & \\
\hline \multicolumn{3}{|l|}{ Gender } \\
\hline Male & 0.52 & $1.4(0.5-3.7)$ \\
\hline Female & & \\
\hline \multicolumn{3}{|l|}{ Localization } \\
\hline Colon & 0.76 & $1.6(0.6-4.2)$ \\
\hline Rectum & & \\
\hline \multicolumn{3}{|c|}{ Performance status WHO } \\
\hline 0 & 0.81 & $0.9(0.3-2.8)$ \\
\hline $1+2$ & & \\
\hline \multicolumn{3}{|c|}{ Adjuvant therapy } \\
\hline No & 0.84 & $0.9(0.2-3.4)$ \\
\hline Yes & & \\
\hline Age (y) & 0.60 & $1.01(0.96-1.07)$ \\
\hline TIMP-1 $\log _{e}$ & 0.001 & $3.6(1.4-9.5)$ \\
\hline
\end{tabular}

TIMP-1 above the median. The results of a multivariate analysis including TIMP-1 and TTP are shown in Table 3. TIMP-1 is not significant at the $5 \%$ level $(P=0.06)$; however, TIMP-1 is the only covariate close to statistical significance and model selection results in TIMP-1 being the only covariate showing statistical significance.

When testing for an association between plasma TIMP-1 scored as a continuous variable on the log scale $\left(\log _{e}\right)$ and OS, a significant association between these two variables was found (HR, 3.8; 95\% CI, 2.4-5.9; $P<0.0001$ ). Kaplan-Meier estimates of OS dichotomizing plasma TIMP-1 by its median level are shown in Fig. 2. As seen from this figure, there was a highly statistically significant difference in OS between patients with low versus high plasma TIMP-1 levels (HR, 4; 95\% CI, 2.2-7.3; $P<0.0001)$. At time point 24 months, 16 patients were still at risk in the low TIMP-1 group whereas only two patients were at risk in the high TIMP-1 group. The survival probability at 24 months was $62 \%$ (95\% CI, 46-77\%) for patients with plasma TIMP-1 below the median level and 7\% (95\% CI, 0-17\%) for those with TIMP-1 levels above the median. Multivariate analysis showed (Table 4) that plasma TIMP-1 was significant

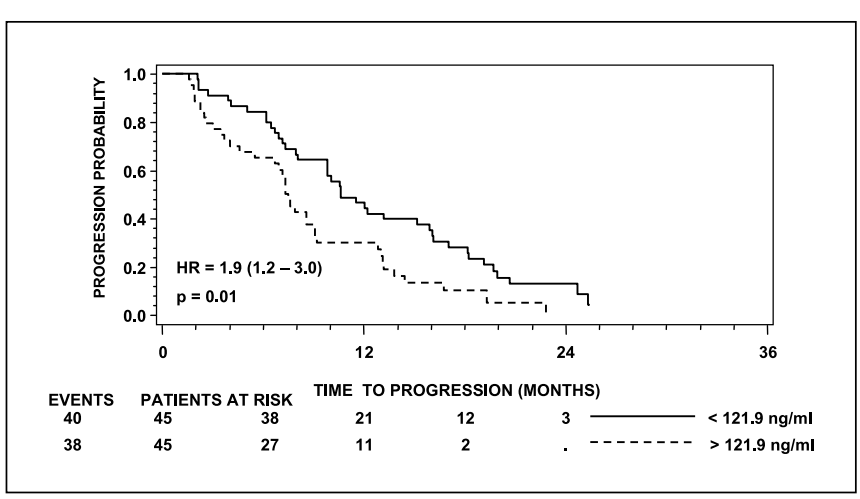

Fig. 1. Kaplan-Meier curves showing the association between plasmaTIMP-1 values and TTP. The number of events (progressions) and number of patients at risk at different time points during the observation period for each of the two groups are given below the figure. Solid line, patients with plasmaTIMP-1 levels below the median; broken line, patients with plasma TIMP-1 levels above the median. 
for OS when including routine clinical baseline covariates (HR, 3.5; 95\% CI, 2.1-5.8; $P<0.0001$ ). The only other significant covariate for OS was performance score (HR, 2.4; 95\% CI, 1.2-4.5; $P=0.008)$. In a multivariable analysis now including TTP instead of OS, plasma TIMP-1 and baseline covariates showed that only plasma log TIMP-1 treated as a continuous variable was retained in the model (HR, 1.5; 95\% CI, 1.0-2.3; $P=0.049$ ).

Assessment of CEA used as a continuous variable on the log scale for the analysis of TTP and OS showed that this model formulation was inappropriate (not linear on a log scale). CEA was not a significant predictor of TTP $(n=68, P=0.56)$ using the conventional cutpoint of 5 units $/ \mathrm{mL}$. Nor was CEA a significant predictor of OS $(P=0.56)$ in a model also including TIMP-1.

\section{Discussion}

The present study shows that high plasma levels of TIMP-1 in patients with metastatic colorectal cancer treated with first-line combination chemotherapy are indicative of low probability of objective response to chemotherapy. This decreased probability of obtaining an objective response to chemotherapy in plasma high TIMP-1 patients was reflected in a significantly decreased TTP and OS of these patients. Based on the present results, it is, however, not possible to clearly separate the effect of TIMP-1 on prognosis $(6-12)$ or on objective response to chemotherapy.

The possibility to predict response to chemotherapy in metastatic colorectal cancer has wide implications as it is anticipated that presently only $\sim 50 \%$ of metastatic colorectal cancer patients receiving chemotherapy have clear benefit thereof. A predictive biomarker could thus be used to protect patients from ineffective treatment and thereby from the adverse effects induced by chemotherapy. On the other hand, the predictive biomarker could be used to select metastatic colorectal cancer patients not only for routine systemic chemotherapy, but also for clinical trials testing either different or more aggressive treatment schedules, or new types of chemotherapy/biological-based therapy.

In the present study, the patients received irinotecan and 5$\mathrm{FU}+$ folinic acid by two different schedules. The treatment

Table 3. Multivariate Cox regression analysis of TTP

\begin{tabular}{|c|c|c|}
\hline Covariate & $\boldsymbol{P}$ & HR (95\% CI) \\
\hline $\begin{array}{l}\text { Randomized } \\
\text { FLIRI } \\
\text { FOLFIRI }\end{array}$ & 0.33 & $1.3(0.8-2.1)$ \\
\hline $\begin{array}{l}\text { Gender } \\
\text { Male } \\
\text { Female }\end{array}$ & 0.33 & $0.8(0.5-1.3)$ \\
\hline $\begin{array}{l}\text { Localization } \\
\text { Colon } \\
\text { Rectum }\end{array}$ & 0.20 & $0.7(0.4-1.2)$ \\
\hline $\begin{array}{l}\text { Performance } \\
\quad 0 \\
1+2\end{array}$ & 0.68 & $1.1(0.6-2.0)$ \\
\hline $\begin{array}{l}\text { Adjuvant the } \\
\text { No } \\
\text { Yes }\end{array}$ & 0.54 & $1.3(0.6-2.8)$ \\
\hline $\begin{array}{l}\text { Age }(y) \\
\text { TIMP-1 } \log _{e}\end{array}$ & $\begin{array}{l}0.31 \\
0.06\end{array}$ & $\begin{array}{c}1.00(0.96-1.01) \\
1.5(1.0-2.3)\end{array}$ \\
\hline
\end{tabular}

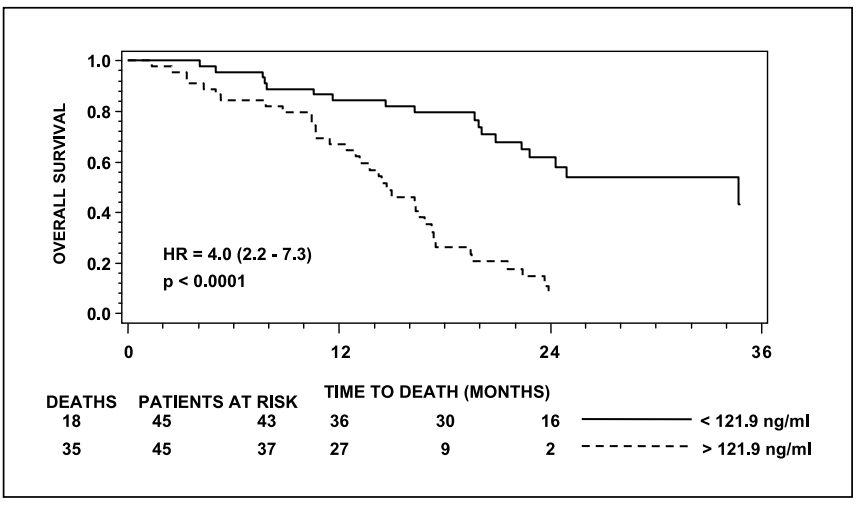

Fig. 2. Kaplan-Meier curves showing the association between plasma TIMP-1 values and OS. The number of events (disease related deaths) and number of patients at risk at different time points during the observation period for each of the two groups are given below the figure. Solid line, patients with plasmaTIMP-1 levels below the median; broken line, patients with plasmaTIMP-1 levels above the median.

schedule was not retained in the multivariate analysis, suggesting that the association between plasma TIMP-1 and objective response was independent of treatment schedule but directly related to tumor sensitivity/resistance to the drugs. Analyses of the entire randomized study of 557 patients reveal that there is no difference between schedules for either TTP or $\mathrm{OS}^{6}{ }^{6}$ The question then arise whether the present results are only valid when these two drugs are being used or whether plasma TIMP-1 would also predict sensitivity/resistance to other classes of chemotherapeutic compounds. We have recently found that patients with metastatic breast cancer and high tumor tissue levels of TIMP-1 in the tumor tissue sampled at the time of primary surgery are resistant to systemic chemotherapy, either the combinations of cyclophosphamide, methotrexate and 5-FU, cyclophosphamide, epirubicin and 5 -FU, or doxorubicin given alone (13). Taking these data together with the present findings suggest that TIMP-1 predicts for sensitivity/resistance to several classes of chemotherapy. The strong associations with both TTP, directly related to the efficacy of the first-line combination, and OS, influenced also by the efficacy of subsequent lines of treatment, suggest that the predictive ability is not restricted to the used combination. In further support of this statement is that in our experimental model consisting of wild-type and TIMP-1 gene-deficient fibrosarcoma cells (16), we consistently find the TIMP-1expressing wild-type cells more resistant than the TIMP-1 genedeficient cells to a variety of chemotherapeutic compounds (etoposide, vincristine, and Cytosar).

CEA levels were also measured in a subset of the patients $(n=67)$. CEA treated as a continuous variable was found to be significantly associated with objective response, with higher serum CEA levels predicting lack of objective response. However, when combining CEA with TIMP-1, no significantly improved fit was obtained. Serum CEA was neither a significant predictor of TTP nor of OS when TIMP-1 was included as the only other covariate.

The antiapoptotic effect of TIMP-1 can be regulated both in a matrix metalloproteinase (MMP)-dependent and a MMPindependent way. In vivo and in vitro experiments, using

\footnotetext{
${ }^{6}$ B. Glimelius et al., unpublished information.
} 
Table 4. Multivariate Cox regression analysis of OS

\begin{tabular}{|c|c|c|}
\hline Covariate & $\boldsymbol{P}$ & HR (95\% CI) \\
\hline $\begin{array}{l}\text { Randomized } \\
\text { FLIRI } \\
\text { FOLFIRI }\end{array}$ & 0.30 & $1.4(0.8-2.4)$ \\
\hline $\begin{array}{l}\text { Gender } \\
\text { Male } \\
\text { Female }\end{array}$ & 0.87 & $0.9(0.5-1.6)$ \\
\hline $\begin{array}{l}\text { Localization } \\
\text { Colon } \\
\text { Rectum }\end{array}$ & 0.74 & $0.7(0.4-1.4)$ \\
\hline $\begin{array}{l}\text { Performance } \\
\quad \begin{array}{l}1+2 \\
1+\end{array}\end{array}$ & 0.008 & $2.4(1.2-4.5)$ \\
\hline $\begin{array}{l}\text { Adjuvant the } \\
\text { No } \\
\text { Yes }\end{array}$ & 0.30 & $1.8(0.6-5.4)$ \\
\hline $\begin{array}{l}\text { Age }(y) \\
\text { TIMP-1 } \log _{e}\end{array}$ & $\begin{aligned} & 0.78 \\
< & 0.0001\end{aligned}$ & $\begin{array}{c}1.00(0.96-1.03) \\
3.5(2.1-5.8)\end{array}$ \\
\hline
\end{tabular}

mammary epithelial cells, have shown that apoptotic effector molecules, such as caspases, are induced by degradation of the extracellular matrix by MMPs, leading to apoptotic cell death $(2-4)$. As such, the anti-MMP function of TIMP-1 would indirectly inhibit apoptosis. In support of this notion is a study of apoptosis in mammary epithelial cells originating from MMP-3 transgenic mice showing that the number of apoptotic cells was significantly reduced when the cells came from MMP-3 transgenic/TIMP-1 - overexpressing mice (4). In support of an MMP-independent TIMP-1 antiapoptotic function is that reduced or alkylated TIMP-1, completely devoid of all MMP inhibitory activity, effectively inhibits apoptosis in Burkitt's lymphoma cells (5). In the same study, it was also reported that addition of recombinant TIMP-1 lead to inhibition of apoptosis, and that addition of anti-TIMP-1 antibodies to neutralize secreted TIMP-1 resulted in a 4 -fold increase in induction of apoptosis. The antiapoptotic mechanism of TIMP-1 could be mediated by interaction with a binding protein, which may induce survival signals through activation of the focal adhesion kinase and/or the phosphatidylinositol 3-kinase, leading to increased expression of the antiapoptotic protein $\mathrm{Bcl}-\mathrm{XL}$ and preventing activation of the caspase cascade $(17-20)$. Indeed, it was recently reported that
TIMP-1 binds to CD63, which is a membrane protein belonging to the family of teraspanin molecules (21). Added TIMP-1 was shown to bind to cell surfaces, inducing activation of the above-described signaling pathway leading to protection of the cells from apoptotic stimuli.

Knowing that TIMP-1 can induce chemoresistance in cancer cells in vitro, it is obvious to speculate whether TIMP-1 could represent a valid target for increasing tumor cell sensitivity to chemotherapy. Normal cells do not overexpress TIMP-1, and the TIMP-1 gene-deficient mouse is without any detectable phenotype, suggesting that systemic inhibition of TIMP-1 in a patient, who subsequently receives chemotherapy, would not induce additional toxicity in the normal tissue. We have recently published that also another proteinase inhibitor, plasminogen activator inhibitor-1, protects cancer cells against apoptosis induced by a wide range of chemotherapeutic compounds $(22,23)$. Of particular interest was that although PAI-1 gene-deficient murine fibrosarcoma cells showed increased sensitivity to chemotherapy in vitro, no difference in systemic toxicity was observed when exposing PAI-1 gene-deficient and wild-type mice to chemotherapy.

The present study was done on plasma. We believe that the elevated plasma TIMP-1 found in the metastatic colorectal cancer patients originates from the metastatic tumor tissue and thereby reflects production and secretion of TIMP-1 from the tumor tissue. It could thus be of interest to test whether tumor tissue TIMP-1 instead of plasma TIMP-1 measurements would give a more direct TIMP-1 tumor measurement and thereby superior results regarding drug sensitivity/resistance. It is noteworthy that the data obtained in our breast cancer study originated from tumor tissue TIMP-1 measurements from the primary tumor (13).

Future studies should be designed to determine clinically relevant cutpoints of plasma TIMP-1 and further prospectively validate plasma TIMP-1 measurements in the prediction of response to chemotherapy in patients with metastatic colorectal cancer. Future studies should also include measurements of other potential predictive markers in addition to TIMP-1 to determine if any other biomarker should be included together with TIMP-1 in a panel of markers to be used to predict sensitivity/resistance to chemotherapy in patients with metastatic colorectal cancer.

\section{References}

1. Benson AB III, Desch CE, Flynn PJ, et al. 2000 update of American Society of Clinical Oncology colorectal cancer surveillance guidelines. J Clin Oncol 2000;18:3586-88.

2. Murphy FR, Issa R, Zhou $X Y$, et al. Inhibition of apoptosis of activated hepatic stellate cells by tissue inhibitor of metalloproteinase- 1 is mediated via effects on matrix metalloproteinase inhibition-implications for reversibility of liver fibrosis. J Biol Chem 2002; 277:11069-76

3. Boudreau N, Sympson CJ, Werb Z, Bissell MJ. Suppression of ice and apoptosis in mammary epithelialcells by extracellular-matrix. Science 1995;267: $891-3$.

4. Alexander CM, Howard EW, Bissell MJ, Werb Z. Rescue of mammary epithelial cell apoptosis and entactin degradation by a tissue inhibitor of metalloproteinases-1 transgene. J Cell Biol 1996;135:1669-77.

5. Guedez L, Stetler-Stevenson WG, Wolff L, et al. In vitro suppression of programmed cell death of
B cells by tissue inhibitor of metalloproteinases-1. J Clin Invest 1998;102:2002-10.

6. Holten-Andersen MN, Murphy G, Nielsen HJ, et al. Quantitation of TIMP-1 in plasma of healthy blood donors and patients with advanced cancer. $\mathrm{Br} J$ Cancer 1999;80:495-503.

7. Holten-Andersen MN, Stephens RW, Nielsen HJ, et al. High preoperative plasma tissue inhibitor of metalloproteinase-1 levels are associated with short survival of patients with colorectal cancer. Clin Cancer Res 2000;6:4292-9.

8. Simpson RA, Hemingway DM, Thompson MM. Plasma TIMP-1 - a marker of metastasis in colorectal cancer. Colorectal Dis 2000;2:100-5.

9. Yukawa N, Yoshikawa T, Akaike M, et al. Plasma concentration of tissue inhibitor of matrix metalloproteinase 1 in patients with colorectal carcinoma. $\mathrm{Br} \mathrm{J}$ Surg 2001;88:1596-601.

10. Holten-Andersen M, Christensen IJ, Nilbert M, et al. Association between preoperative plasma levels of tissue inhibitor of metalloproteinases 1 and rectal cancer patient survival: a validation study. Eur J Cancer 2004:40:64-72.

11. Yukawa N, Yoshikawa T, Akaike M, et al. Prognostic impact of tissue inhibitor of matrix metalloproteinase1 in plasma of patients with colorectal cancer. Anticancer Res 2004;24:2101 - 5.

12. Waas ET, Hendriks T, Lomme RMLM, Wobbes T. Plasma levels of matrix metalloproteinase- 2 and tissue inhibitor of metalloproteinase-1 correlate with disease stage and survival in colorectal cancer patients. Dis Colon Rectum 2005;48:700-10.

13. Schrohl AS, Meijer-van Gelder ME, HoltenAndersen MN, et al. Primary tumour levels of tissue inhibitor of metalloproteinases-1 are predictive of resistance to chemotherapy in patients with metastatic breast cancer. Clin Cancer Res 2006;12: 7054-8.

14. Lipton A, Ali SM, Leitzel K, et al. Elevated plasma TIMP-1 levels predicts decreased response and survival 
in metastatic breast cancer. Breast Cancer Res Treat 2005;94:49.

15. Therasse $P$, Arbuck SG, Eisenhauer EA, et al. New guidelines to evaluate response to treatment in solid tumors. J Natl Cancer Inst 2000;92:205-16.

16. Davidsen ML, Würtz $S \emptyset$, Rømer MU, et al. TIMP-1 gene deficiency increases the sensitivity to chemotherapy-induced apoptosis. Br J Cancer 2006;95: $1114-20$.

17. Li G, Fridman R, Kim HR. Tissue inhibitor of metalloproteinase-1 inhibits apoptosis of human breast epithelial cells. Cancer Res 1999;59:6267-75.

18. Lambert E, Boudot $C$, Kadri Z, et al. Tissue inhibitor of metalloproteinases-1 signalling pathway leading to erythroid cell survival. Biochem J 2003:372:767-74.

19. Liu XW, Bernardo MM, Fridman R, Kim HRC. Tissue inhibitor of metalloproteinase-1 protects human breast epithelial cells against intrinsic apoptotic cell death via the focal adhesion kinase/phosphatidylinositol 3kinase and MAPK signaling pathway. J Biol Chem 2003;278:40364-72.

20. Lee SJ, Yoo HJ, Bae YS, Kim HJ, Lee ST. TIMP-1 inhibits apoptosis in breast carcinoma cells via a pathway involving pertussis toxin-sensitive $\mathrm{G}$ protein and c-Src. Biochem Biophys Res Commun 2003;312: 1196- 201.
21. Jung K-K, Liu X-W, Chirco R, Fridman R, Kim H-R C. Identification of CD63 as a tissue inhibitor of metalloproteinase-1 interacting cell surface protein. EMBO J 2006;25:3934-42.

22. Lademann $U$, Romer MU, Jensen $P B$, et al. Malignant transformation of wild-type but not plasminogen activator inhibitor-1 gene-deficient fibroblasts decreases cellular sensitivity to chemotherapy-mediated apoptosis. Eur J Cancer 2005;41:1095-100.

23. Romer MU, Kirkebjerg DA, Larsen KJ, et al. Indication of a role of plasminogen activator inhibitor type I in protecting murine fibrosarcoma cells against apoptosis. Thromb Haemost 2005;94:859-66. 